\title{
Assessing the Impact of Wind Conditions on Urban Heat Islands in Large Australian Cities
}

\author{
Ilham Al-Obaidi'*, Scott Rayburg², Marek Półrolniczak³ ${ }^{3}$ Melissa Neave ${ }^{4}$ \\ 1 Mustansiriyah University, Iraq \\ 2 Swinburne University of Technology, Melbourne, Australia \\ 3 Adam Mickiewicz University, Poland \\ ${ }^{4}$ Centre for Urban Research, RMIT University, Melbourne, Australia \\ * Corresponding author's e-mail: ialobaidi@swin.edu.au
}

\begin{abstract}
Urban regions are well known to be warmer than the outlying surrounding regions: this phenomenon is termed an Urban Heat Island (UHI). Depending upon its severity, an UHI can influence human health, the condition of urban vegetation, as well as air and water quality leading to a general decline in the living conditions of the affected urban environments and residents. Some studies have shown that prevailing weather conditions, like wind patterns, can influence UHI magnitudes. These studies suggest that wind speeds may be inversely related to UHI magnitude. However, long-term and high frequency weather and temperature measurements are exceedingly rare, so the exact nature of the relationship between wind speeds and directions as well as UHI magnitudes remain unknown. In order to address this problem, this study investigates how UHI magnitudes in five Australian cities affect wind speed and wind direction. The results of this study revealed that urban-non-urban temperature differences are most pronounced under calm weather conditions. The UHI intensity weakened as wind velocity increased: strong significant negative correlations were found between the mean UHI intensity and mean wind speed magnitudes. The results show that the greatest UHI intensities are recorded when wind is weak (less than $2 \mathrm{~ms}^{-1}$ ), while the lowest magnitudes are found when wind speeds exceed $6 \mathrm{~ms}^{-1}$. Further, the results show that the critical wind speed value, above which the strength of the UHI is considerably minimized, is around $4-5 \mathrm{~ms}^{-1}$. In addition, the study shows that wind direction in each city is a critical driver factor that determines the intensity of the UHI effect. When winds originate from dry environments, they favour high UHI intensities at all wind speeds, while the winds from the ocean side of coastal cities tend to cool urban regions, reducing UHI intensities or even promoting the urban cool island formation.
\end{abstract}

Keywords: climate change, heat islands, wind speed and wind direction, seasonal and diurnal UHI

\section{INTRODUCTION}

Urban Heat Islands (UHIs), where urban areas are warmer than the surrounding rural areas, are a common and growing phenomenon in cities. These features are a direct consequence of urbanisation, which removes natural vegetation and water bodies, and replaces them with low albedo impervious surfaces. The importance of UHIs and how to mitigate them is growing, as the effects of climate change and UHIs can combine to make cities less liveable with increasing heat stress a common occurrence in many of the world's cities.
Indeed, it is estimated that the risk of heat-related hospital admissions in urban regions is about double that of surrounding areas (Knowlton et al. 2007). For example, in Melbourne, it is known that the heat-related hospital callouts increase significantly when temperatures exceed $30^{\circ} \mathrm{C}$. This will occur much more frequently in urban areas on account of UHIs than it would have under natural conditions. One major contributor to the heat related illnesses and hospital visits is the heat that does not dissipate at night (Kenney et al., 2014)

The urban heat island effect refers to the warming of the urban canopy layer (UCL) and 
urban boundary layer (UBL) relative to the neighbouring rural sites. Several studies indicated that the urban heat island phenomenon is created by the thermo-physical characteristics of built up urban areas (e.g., impervious and dark surfaces), building patterns (e.g., proximity of buildings to one another and building orientation), as well as anthropogenic and neighbouring heat sources (Zhong et al., 2017; Prafull et al., 2017; Shashua et al., 2000; Oke 1982).The phenomenon may occur during day or night time periods, whereas its temporal and spatial form is controlled by the unique features of each urban area; however, it is often the highest overnight (Santamouris, 2015; Harlan et al., 2006).

UHIs are considered a global problem and many researchers, meteorologists and engineers have been worked on understanding and discovering the causes and effects of this phenomenon. This has led to greater knowledge of UHIs as well as adaption and mitigation methods. The research into meteorological factors suggests that wind speed and cloudiness are significant meteorological parameters in terms of their impact on UHI intensity, as they affect long wave radiation losses and ventilation (Kim \& Baik, 2004; Morris \& Simmonds, 2000). Considerable research into the effect of these parameters has revealed that the urban-rural temperature differences are most pronounced under calm and clear atmospheric conditions ( $\mathrm{Li}$ et al., 2011; Morris et al., 2001; Yong-Jiang, 2009). For example, YongJiang (2009) found that strong winds facilitate the mixing of air, thus reducing the temperature differences between urban and non-urban sites. In addition, cloudless conditions resulted in lower urban-non-urban temperature differences because the night-time cooling processes were more effective when there were no clouds to reradiate the long wave energy back to the ground surface. Sani (1973) also reported that the urban heat island intensity ranged between $2.0^{\circ} \mathrm{C}$ to $2.2^{\circ} \mathrm{C}$ on cloudy days but was between $4.5^{\circ} \mathrm{C}$ to $5.0^{\circ} \mathrm{C}$ on clear days. Working on the data from Melbourne, Morris et al. (2001) observed that the UHI intensity was higher under clear and calm conditions (maximum UHI magnitude was $5.0-6.0^{\circ} \mathrm{C}$ under calm conditions with wind speeds lower than $2 \mathrm{~m} / \mathrm{s}$ ), whereas when wind speeds exceeded $5 \mathrm{~m} / \mathrm{s}$ the UHI intensity was observed to be low. Erell and Williamson (2007) showed that the UHI intensity in Adelaide weakened as wind velocity increased. Although the relationship between wind direction, wind speed and UHI intensity has been relatively well established, not all studies into the impacts of wind data have returned clear evidence of its impact. For example, the research by Sofer and Potchter (2006) in Israel and Elsayed (2011) in Malaysia showed no significant increase in UHI intensity under calm conditions, suggesting that cooling from wind was not an important control in these environments.

A reason for the disparity between results in this topic may stem from the limited range of conditions under which observations have been made and points to the need for further research into this topic. For example, most UHI investigations compare one rural site to one urban site to calculate UHI intensity, typically over a relatively short time frame and in one city only, while neglecting the impact of other meteorological parameters, like wind speeds and directions. In order to address this problem, this study has collected the largest temporal and spatial data set on UHI thus far considered in the literature. These data include hourly climate observations over 19 years from one urban observation station and one rural observation station in each of five Australian cities (Sydney, Melbourne, Adelaide, Canberra and Brisbane) to study the impact of both of the wind speed and wind direction on UHI intensity. The cities are located in disparate geographic regions, each with a unique urban configuration and differing climate characteristics.

\section{METHOD AND DATA PROCESS}

For each selected site in each investigated city, the hourly air temperature recordings were collected from the Bureau of Meteorology's (Australia) observatory database. These included the observations over a time period approximately of 19 years for most study sites in the five Australian cities (each data file contained more than 200,000 hourly observations per site). However, for Canberra, the data was only available for the last 7 years (2008-2015). In each city, the metrological observation stations were positioned in one urban region and one rural region. The stations were chosen based on the characteristics of the surrounding landscape and the number of impervious surfaces in comparison to natural surfaces, as noted by Oke et al. (2006). These stations have a sufficiently long history to track changes 
in UHI intensity over time and to ensure that the results are robust and not sensitive to short term climatic variations. In addition, the sites were selected to minimize the effects of elevation as this factor alone can result in differences in temperature between sites. The difference in elevation between all study sites in each city is always less than $100 \mathrm{~m}$ (and typically less than $30 \mathrm{~m}$ ). Furthermore, the quality of the meteorological observations was checked against the international observing standards of the World Meteorological Organization. This is the first study to investigate the relationships between weather parameters and UHI intensity for hourly data across a long time frame for multiple cities. This study presents a novel method for relating wind speed and direction to UHI intensity to facilitate the analysis. This involves using polar plots and wind roses colour coded with UHI intensities to illustrate which wind speeds and directions correspond to high or low UHI intensities. The analysis of variance and regression techniques were also used to explore the relationships between weather parameters and UHI intensity and to predict the behaviour of the UHI in numerical terms for mean diurnal, seasonal and annual cycles.

Once collected, the data sets were analysed and checked for irregularities or inconsistencies, the clearly incorrect data were removed (e.g., where the temperature value was entered incorrectly). The research work is based on several statistical tests, including linear regression analysis, as well as wind and UHI rose analyses, using the $\mathrm{R}$ program and its packages: stats (R Core Team, 2019), dplyr (Wickham et al., 2021), and openair (Carslaw and Robkins, 2012). As this study is focused on the impact of wind data (wind speed and wind direction) on the UHI effect, the first step in the analysis was to turn the temperature data into UHI data. This involved creating differences series of temperatures where the urban site temperatures were subtracted from the rural site temperatures (so that when the urban temperatures exceeded the rural temperatures, a positive UHI value is recorded and when the rural temperatures exceeded the urban temperatures, a negative UHI or UCI was recorded).

Next, for the analysis of the relationship between the wind data and UHI intensity, the data were explored for day, night and seasonal cycles, using the wind data retrieved from the surface weather stations. The temporal determination of the wind data conditions is hourly, concurring with the temporal determination of the air temperature data. The first part of analysis (Wind and UHI Rose Analysis) deals with generating the UHI polar plot and wind roses for the selected sites scattered across each city. The UHI rose gives the strength of the wind direction on the UHI effect. In other words, it identifies which direction is associated with strong and weak UHI intensities. The UHI rose plots have been drawn annually, daily and seasonally for the selected stations in each city. The third part of the analysis deals with linear regression analysis between the mean UHI intensity and wind speed for each urban density site for all study cities.

\section{Climate in the study cities}

Australia has a highly diverse climate (Qureshi \& Whitten 2014) as it is driven by an array of large-scale climatic phenomenon. Chief amongst these is the El Nino-Southern Oscillation (Khan et al. 2013) with the second most important being the Indian Ocean Dipole. In addition to these, both climate change and other human activities are making the Australian climate even more variable and unpredictable. This study examines the impact of wind data on urban heat island effect in five Australian cities. These are Melbourne, Sydney, Canberra, Adelaide and Brisbane. The cities are located in disparate geographic regions; each has a unique urban configuration and differing climate characteristics. Some basic properties of each of these cities (that highlights these differences) are presented in Table 1.

\section{Descriptions of the meteorological stations}

Table 2 presents the characteristics of the meteorological stations in the urban and rural sites selected in each city.

\section{RESULTS}

This study considered how wind speeds and directions influence UHIs using three approaches. First, these relationships were explored over the diurnal cycle. Next, the relationships were explored using a seasonal lens. Finally, regression analyses were used to derive the relationships between UHI intensity as well as wind speed and wind direction over the entire data set. 
Table 1. Summary of the population and climatic character of each of the five cities included in this study

\begin{tabular}{|c|c|c|}
\hline City & $\begin{array}{c}\text { Population } \\
\text { (Growth rate 2011-2017) }\end{array}$ & Climatic character \\
\hline Melbourne & $\begin{array}{l}4.82 \text { million } \\
(3.22 \%)\end{array}$ & $\begin{array}{l}\text { Temperate climate with plenty of sunshine. The temperature regularly exceeds } 35^{\circ} \mathrm{C} \text {. } \\
\text { Wind speed and direction vary during the day and night and seasonally. Wind speed } \\
\text { is typically lowest overnight and in the early morning. Wind speed then rises during } \\
\text { the day and into the afternoon. There is also a marked tendency for very windy days } \\
\text { to occur during the summer and spring. }\end{array}$ \\
\hline Sydney & $\begin{array}{l}5.57 \text { million } \\
(6.15 \%)\end{array}$ & $\begin{array}{l}\text { Humid subtropical climate with abundant sunshine. The maximum air temperature } \\
\text { was } 46^{\circ} \mathrm{C} \text { in January } 2013 \text {. Climate tends to be general colder in the south and } \\
\text { warmer moving to the north. Summer is hot with warm nights. Winters are cool and } \\
\text { rainy. The annual dominant wind direction is north easterly, with the average wind } \\
\text { speed equal to } 13.4 \mathrm{~km} / \mathrm{h} \text {. }\end{array}$ \\
\hline Canberra & $\begin{array}{l}396,785 \\
(1.50 \%)\end{array}$ & $\begin{array}{l}\text { Relatively dry, continental climate with warm to hot summers and cool to cold } \\
\text { winters. In summer, the city experiences dry westerly and northerly winds, whilst in } \\
\text { winter the city receives more cold southerly winds, with average wind speeds on the } \\
\text { order of } 13.5 \mathrm{~km} / \mathrm{h} \text {. }\end{array}$ \\
\hline Adelaide & $\begin{array}{l}1.37 \text { million } \\
(2.25 \%)\end{array}$ & $\begin{array}{l}\text { Mediterranean climate. Summers are hot with temperatures regularly exceeding } \\
40^{\circ} \mathrm{C} \text {. In winter, temperatures range between } 8^{\circ} \mathrm{C} \text { and } 16^{\circ} \mathrm{C} \text {. The wind speed } \\
\text { direction vary diurnally and seasonally, with southerly winds common in spring and } \\
\text { summer (during the afternoon).Morning period winds are generally more northerly } \\
\text { outside of the hotter months. }\end{array}$ \\
\hline Brisbane & $\begin{array}{l}2.47 \text { million } \\
(3.65 \%)\end{array}$ & $\begin{array}{l}\text { Sub-tropical climate with hot weather for most of the year. In summer, the humidity } \\
\text { can become trying at times. Winter time is dry and mild. In winter, prevailing winds } \\
\text { in Brisbane are from the south, whilst from November to February; prevailing winds } \\
\text { come from the north. }\end{array}$ \\
\hline
\end{tabular}

Table 2. The characteristics of the meteorological stations

\begin{tabular}{|c|c|c|c|c|c|c|}
\hline City & Weather station & Elevation $(\mathrm{m})$ & Resolution & Data Period & Urbanisation level & Description \\
\hline \multirow[t]{2}{*}{ Melbourne } & $\begin{array}{c}\text { Melbourne } \\
\text { Regional Office }\end{array}$ & 31.0 & Hourly data & $1997-2014$ & Urban site & High density and high traffic \\
\hline & Coldstream & 83.0 & Hourly data & 1996-2015 & Rural site & Forest and vegetation \\
\hline \multirow{2}{*}{ Sydney } & Sydney Airport & 6.0 & Hourly data & $1996-2015$ & Urban site & $\begin{array}{l}\text { Medium- density urban and very } \\
\text { high traffic }\end{array}$ \\
\hline & $\begin{array}{l}\text { Richmond } \\
\text { RAAF }\end{array}$ & 19.0 & Hourly data & 1996-2015 & Rural site & Vegetation and pervious ground \\
\hline \multirow[b]{2}{*}{ Adelaide } & Kent Town & 48.0 & Hourly data & $1997-2014$ & Urban site & Dense city buildings and structures \\
\hline & $\begin{array}{l}\text { Roseworthy } \\
\text { Awe }\end{array}$ & 65.0 & Hourly data & 1997-2014 & Rural site & vegetation and pervious ground \\
\hline \multirow{2}{*}{ Canberra } & Tuggeranong & 586.7 & Hourly data & 2008-2015 & Urban site & $\begin{array}{l}\text { Residential neighbourhood with } \\
\text { medium-density, medium traffic, } \\
\text { low vegetation cover }\end{array}$ \\
\hline & $\begin{array}{l}\text { Braidwood } \\
\text { Racecourse } \\
\text { Aws }\end{array}$ & 665.2 & Hourly data & 2008-2015 & Rural site & $\begin{array}{c}\text { Far from the city centre, open area } \\
\text { (pure vegetation area) }\end{array}$ \\
\hline \multirow{2}{*}{ Brisbane } & Brisbane & 8.13 & Hourly data & 1999-2015 & Urban site & $\begin{array}{l}\text { Residential neighbourhood with } \\
\text { medium density buildings, high } \\
\text { traffic, low vegetation cover }\end{array}$ \\
\hline & Amberley Amo & 24.2 & Hourly data & 1999-2015 & Rural site & $\begin{array}{c}\text { Open area, large pervious ground } \\
\text { cover (bare soil, and vegetated } \\
\text { areas) }\end{array}$ \\
\hline
\end{tabular}

\section{The association between wind data and the urban heat island intensity throughout the diurnal cycle}

The results of the statistical analysis for the diurnal data are depicted in Figures 1 to 4 . These data present polar plots and wind roses that show the association of UHI with wind speed and wind direction for the various Australian cities. In general, wind speed negatively associates with urban heat formation with calm winds more likely to facilitate the formation of a strong UHI.The highest UHI intensities were observed at the urban site under calm conditions. It can be seen (Figure 1a) that the maximum average value of UHI was $4.0^{\circ} \mathrm{C}$ to $5.0^{\circ} \mathrm{C}$ which occurred under calm to low wind speed conditions (approximately ranging from $0 \mathrm{~m} \cdot \mathrm{s}^{-1}$ to $2 \mathrm{~m} \cdot \mathrm{s}^{-1}$ ) and as wind speed increases, the UHI magnitude decreases. The lowest UHI intensities (which were negative, so 
representing urban cool island UCIs) were found to be less than $-2.0^{\circ} \mathrm{C}$ at a wind speed above $5.0 \mathrm{~m} \cdot \mathrm{s}^{-1}$ and when winds blow from the south, southeast or southwest. In contrast, the intensities of UHIs are relatively higher when winds originate from the east and northeast directions. Moreover, it is evident in the wind rose plot (Figure 1b) that the prevailing average wind direction is from the northeast (approximately 26\% of the time). However, for the city of Sydney, the results indicate that the UHI intensity is gradually reduced as wind speed increases and is lowest for the wind speeds higher than approximately $7 \mathrm{~m} \cdot \mathrm{s}^{-1}$.

A detailed analysis (Figure 1c, d) shows that for the urban site, high positive UHI intensities were observed only when the air flow is from the inland (northwest) and when wind speed ranged between 0 to $6 \mathrm{~m} \cdot \mathrm{s}^{-1}$. Meanwhile, the lowest or negative UHIs (also known UCIs) were found during calm weather conditions when winds blew from the northeast or southeast (ocean side of the city). Similar results were found in the city of Brisbane. These results show that the UHI intensity is inversely related to wind speed. This, however, may also depend on wind direction. It was found that the UHI intensities are greatest $\left(3-4^{\circ} \mathrm{C}\right)$ for calm to light wind speeds, and as wind speed increases the UHI intensity decreases. Meanwhile, the negative UHI intensities (UCIs) occur when winds blow from the ocean side (northeast, northwest or east) and reached their peak during strong winds. In contrast, the winds from the southwest or northwest (inland side) tend to yield higher positive UHI intensities. However, in the city of Adelaide, it was observed that strong UHI intensity corresponded to north-easterly winds (Figure $1 \mathrm{~g}, \mathrm{~h}$ ). The positive UHI intensities were observed only when the air originates from the mainland side (north, northeast or east) for all wind speeds. The maximum positive UHI intensities for this site occur at wind speeds between 3 to $5 \mathrm{~m} \cdot \mathrm{s}^{-1}$. Negative UCIs occur when winds originate from the south, west, southeast or southwest directions under low to high wind speeds and reached their peak under strong wind conditions. The last city examined was Canberra (Figure 1i, j). The lower UHI intensities were found there under calm wind speeds, and the UHI intensity increases along with wind speed. This contrasts with all of the other cities. The highest UHI intensities $\left(>5^{\circ} \mathrm{C}\right)$ occur when the winds blow from the east or northeast at wind speeds $>3 \mathrm{~m} \cdot \mathrm{s}^{-1}$. In contrast, lower or negative UHI intensities appear when air flow comes from the west and northwest.

Next, the data were partitioned into daytime and night time datasets to examine the day - night dependency of UHI intensity on the wind conditions. In general, there was a clear influence of the wind speed and wind direction on day and night time UHI intensities. For all observed cases, lower UHI intensities were found for calm wind speeds for both day and night periods. In addition, UHI intensities increase along with wind speed for both day and night periods. However, these patterns are also influenced by wind direction. The highest frequency of strong positive UHIs occur overnight, but these are distributed across a variety of wind speeds, while the highest intensity UHIs during the day all occur at very low wind speeds. The data also show that night times have a wider distribution of wind speeds than during the day. This suggests that, in all study cities, stronger winds are experienced, on average, overnight than during the day. However, in all cases, the daytime UHI is typically higher than the night time UHI under calm conditions. The highest day time and night timeUHI intensities in the city of Melbourne are associated with light winds, that is clear in the east, east-north or east southern wind direction. Conversely, negative UHI intensities (UCIs) were observed more frequently at night time than at day time, especially when wind is blowing from the west or south direction. In contrast, for the city of Sydney and Brisbane, the wind from northwest are mainly resulted on a maximum day time and night time UHI, whereas, the wind from ocean side (eastern direction) were observed to be responsible for the reduction

Table 3. Seasonal average UHI intensity for all study cities under calm condition (wind speed = zero)

\begin{tabular}{|l|c|c|c|c|c|}
\hline \multicolumn{1}{|c|}{ Study cities } & Melbourne & Sydney & Adelaide & Canberra & Brisbane \\
\hline Season & $\mathrm{UHI}\left({ }^{\circ} \mathrm{C}\right)$ & $\mathrm{UHI}\left({ }^{\circ} \mathrm{C}\right)$ & $\mathrm{UHI}\left({ }^{\circ} \mathrm{C}\right)$ & $\mathrm{UHI}\left({ }^{\circ} \mathrm{C}\right)$ & $\mathrm{UHI}\left({ }^{\circ} \mathrm{C}\right)$ \\
\hline Summer & 3.56 & 2.07 & 2.26 & 1.75 & 1.23 \\
\hline Autumn & 4.30 & 3.00 & 1.30 & 0.37 & 1.69 \\
\hline Winter & 3.91 & 3.46 & 1.62 & -0.47 & 2.39 \\
\hline Spring & 3.93 & 2.76 & 3.25 & 0.5 & 2.01 \\
\hline
\end{tabular}



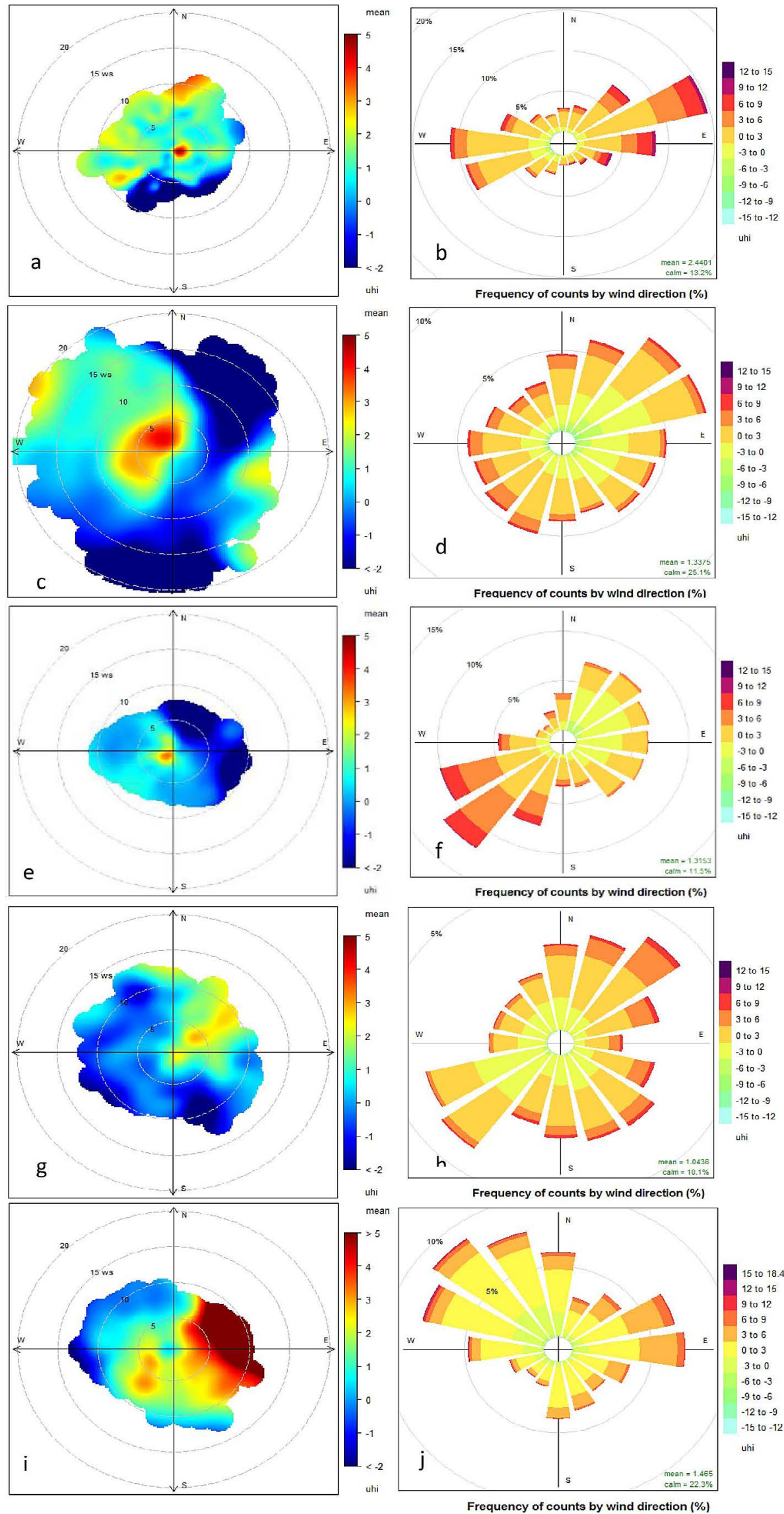

Figure 1. Polar plots and wind roses showing the association of UHI intensity with wind speed and wind direction at different characteristics of Australian cities ( $a$ and $b$ : Melbourne; $\mathrm{c}$ and $\mathrm{d}$ : Sydney; e and f: for of Brisbane; $\mathrm{g}$ and $\mathrm{h}$ : Adelaide; and $\mathrm{i}$ and $\mathrm{j}$ : Canberra) 

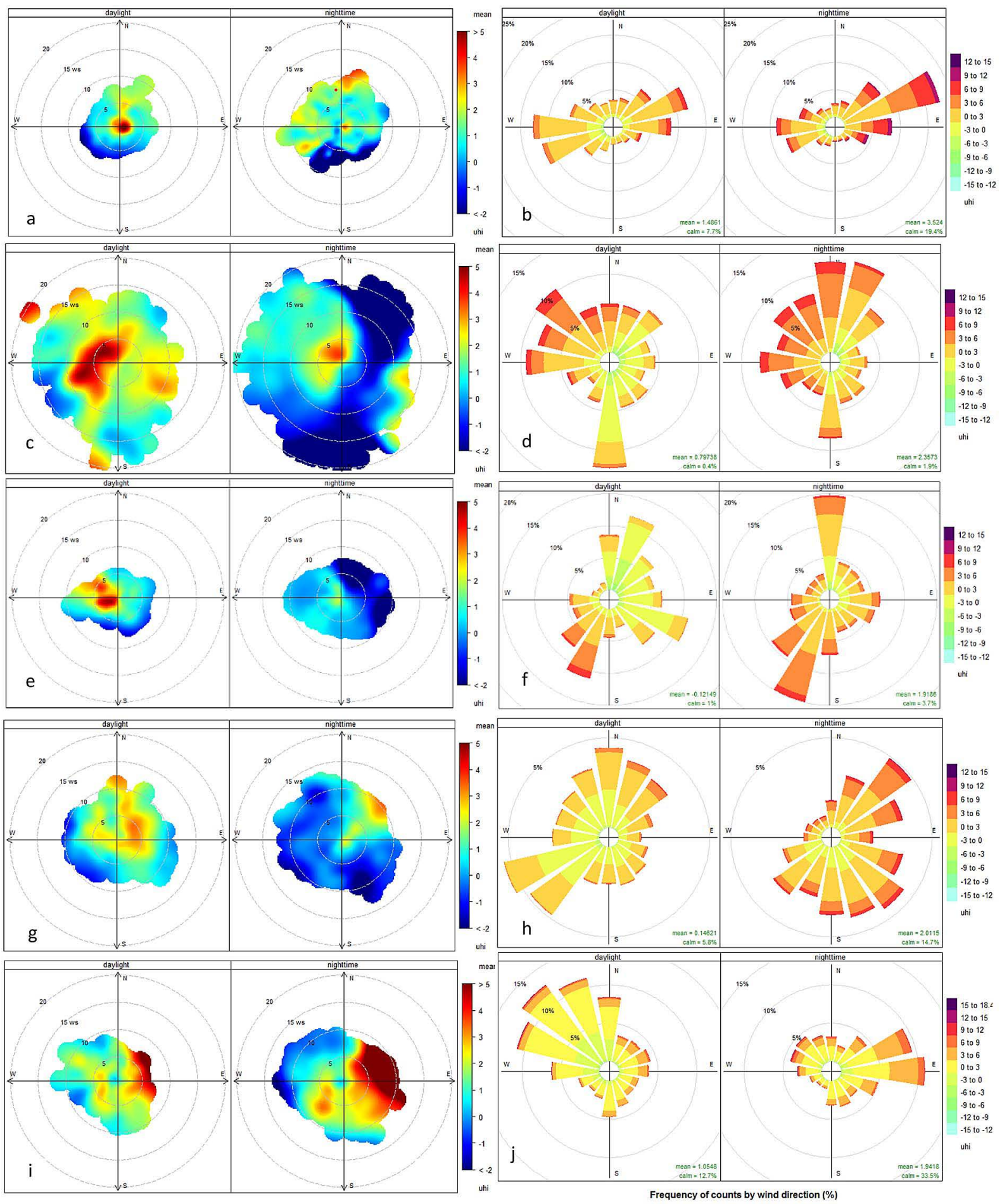

Figure 2. The association of day and night time UHI intensity with the wind speed and wind direction during the diurnal cycle at different characteristics of Australian cities (a and b: Melbourne; $\mathrm{c}$ and $\mathrm{d}$ : Sydney; e and f: for of Brisbane; $\mathrm{g}$ and h: Adelaide; and i and j: Canberra)

the UHI intensities. In the city of Adelaide, high positive nocturnal UHI intensities are associated with the wind blowing from the northeast at calm to high wind speed conditions, while lower or negative nocturnal UHI intensities (UCIs) are correlated with the wind blowing from all directions (except the northeast or east) under low to high wind speed conditions. On the other hand, positive daytime UHI intensities occurred under all wind conditions but reached their peak when winds flowing from the mainland side (north, northeast and east). At wind speeds $>7 \mathrm{~m} \cdot \mathrm{s}^{-1}$, the daytime UCIs were found only when winds blew from the ocean side (west or southwest). The last 
diurnal analysis has been done for the city of Canberra. The strongest daytime and nighttime UHI intensities occur only when winds are blowing from the east, southeast or northeast. Conversely, negative UHI intensities (UCIs)occur more frequently at night than during the day, particularly when winds blow from the west at high wind speeds (wind speeds $>10 \mathrm{~m} \cdot \mathrm{s}^{-1}$ ).

\section{The association between wind conditions and urban heat island intensity throughout the seasonal cycle}

In order to better comprehend the relationship between wind speed, wind direction and UHI intensity, the data were also broken down to consider seasonal patterns for all study cities over the study period. These results are plotted in Figures 3 and 4. These data show that the higher UHI intensities are associated with lower wind speeds in all seasons for all study cities. However, wind direction also influences UHI intensities. Table 3 depicts the seasonal average UHI intensity under calm conditions (wind speed = zero) for all study cities. The highest UHI intensities were observed during the cold winter period, followed by autumn, spring and summer which had the lowest average UHI intensities that were clear in Melbourne, Sydney and Brisbane.

For Adelaide (Canberra) the results exhibit considerable seasonal variation, with the highest UHI intensities observed in spring (summer), followed by summer (spring), winter (autumn) and autumn (winter). Figure 3 shows the effect of wind direction and wind speed on UHIs in different seasons. As depicted in Figure $3(\mathrm{a}, \mathrm{b})$ for the urban region in the city of Melbourne, in all seasons the highest UHI intensities corresponding to the winds that originate in the northwest or western wind directions (inland region) at wind speed of about 0 to $5 \mathrm{~m} \cdot \mathrm{s}^{-1}$. Meanwhile, UCIs are observed only when the wind speed is from the sea direction (south, southeast or northeast) with wind speeds above $5 \mathrm{~m} \cdot \mathrm{s}^{-1}$ in the summer and spring seasons or above $10 \mathrm{~m} \cdot \mathrm{s}^{-1}$ in the cold seasons (winter and autumn). For both Sydney and Brisbane, the UHI intensity decreases with increasing wind speed in all seasons. Figure $(3 \mathrm{c}$,
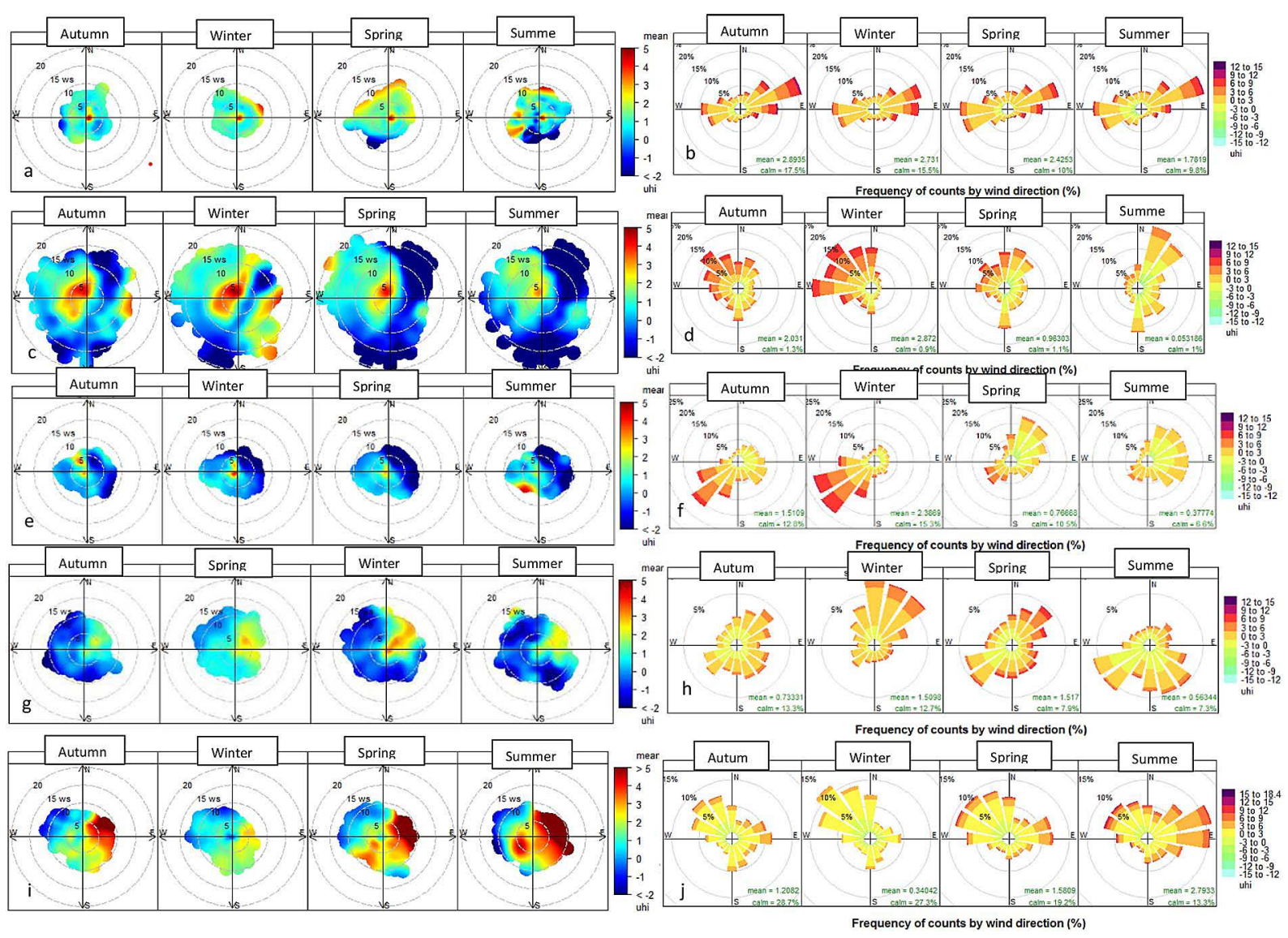

Figure 3. The effect of wind direction and wind speed on the UHIs in different seasons (a and b: Melbourne; c and d: Sydney; e and f: for of Brisbane; $g$ and h: Adelaide; and i and j: Canberra) 
d), the highest UHI intensities are associated with lower wind speeds (wind speeds $<2 \mathrm{~m} \cdot \mathrm{s}^{-1}$ ). The winds that originate from the southwest, northwest or west yield higher UHI intensities, while the winds from the northeast, east or southeast result in higher UCI intensities in all seasons. The prevailing winter winds are mainly from the west and southwest. However, in the city of Sydney, the prevailing winds are mainly from the northeast and north orientation, which is resulting in higher UHI (Figure 3e, f). In contrast, maximum UHI intensities tend to occur only when winds blow from the northeast or east directions (inland region) for all wind speed conditions for Adelaide's urban sites. Figure $(3 g, h)$ shows maximum UCI intensities are frequently found in spring and summer when winds originate from the southeast, southwest or northwest. Canberra's urban site (Figure $3 \mathrm{i}, \mathrm{j}$ ) shows different pattern, lower positive UHI intensities were recorded under low wind speed conditions (wind speed $<2 \mathrm{~m} \cdot \mathrm{s}^{-1}$ ). For wind speeds $>2 \mathrm{~m} \cdot \mathrm{s}^{-1}$, the urban region exhibits a positive UHI intensities in all seasons. The strongest UHI intensities $\left(>5^{\circ} \mathrm{C}\right)$ were observed only when winds came from the northeast or east at wind speeds $>5 \mathrm{~m} \mathrm{~s}^{-1}$ in all seasons. Southeast winds were also related to higher UHI intensities at high wind speeds, particularly during spring and summer, while the winds from the northwest or west tend to yield lower or negative UHI intensities at strong wind speeds, particularly during summer and autumn. The predominant summer wind directions are mainly from the east and northeast, whereas the prevailing winter wind directions are mainly from the northwest and north.

Next, the seasonal data was broken down into day time and night time components. These results are presented in Figure 4. Generally speaking, the negative correlations between winds speed and UHI intensities existed for both day and night across all seasons. However, the results suggest that the mean daytime UHI intensities are generally higher than the mean night time UHIs under calm or low wind speed conditions during all seasons in all cases. Observation of night time and daytime Melbourne's UHI intensities as well as wind speeds and wind directions for the urban site (Figure 4a, b) show that greater UHI intensities occur under calm to light wind speeds when winds blow from the east or northeast during all seasons. For wind speeds above $2 \mathrm{~m} \cdot \mathrm{s}^{-1}$, the nocturnal UHI intensity decreases as wind speed increases, but during daytime UHI intensities tended to be lower at wind speeds above $5 \mathrm{~m} \cdot \mathrm{s}^{-1}$ during all seasons. The prevailing wind direction is from the northeast during the night time in all seasons and during the day time in winter and autumn but shifts to the west and southwest during the spring and summer during the day. The day time UHI intensity tended to be highest in autumn and lowest in winter under calm conditions. Meanwhile, the greatest night time UHI intensities were observed in winter and the weakest were found in summer. In contrast, the UCI effect was more frequent during the day period than the night period, especially in hot seasons (spring and summer). During the spring season, only wind speeds greater than $7 \mathrm{~m} \cdot \mathrm{s}^{-1}$ and originating from the southwest yield UCIs, whereas the UCI effect was rarely observed during the cold seasons (autumn and winter). The results also show a negative correlation between wind speed and Sydney's UHI intensities in all seasons, especially at night (Figure 4c, d). However, the strongest daytime and night time UHI intensities occurred in winter, followed by autumn, and were weakest in summer for most study sites. It has been seen that the mean daytime UHIs are generally higher than the mean night time UHIs under calm or low wind speed conditions during all seasons. Observations of night time and daytime UHI intensities and wind speed and wind direction for urban site indicate that high positive daytime UHI intensities were observed under low to high wind speeds when the wind blew from the west or northwest during all seasons (except in winter, where all wind directions can yield high positive UHI intensities). The maximum negative UHI intensities are mainly recorded in summer and spring when airflow is from the ocean side at strong wind speeds. In contrast, the night time UHI intensities tend to be highest under low to calm wind speed conditions, however as wind increases, the UHI decreases in all seasons. It is evident that the wind from western or northwestern directions (inland side) gives positive UHI values, whereas, the wind from the ocean side (northeast, east, south or southeast directions) gives negative UHI intensities for light wind speeds and UCIs at high wind speeds. In the winter and autumn seasons, the prevailing wind is from the northwest and west direction (inland side), while in summer, a very significant part of the wind originates from the northeast and south for both day and night periods (Figure 4d). On the other hand, in city of Brisbane, it was found 


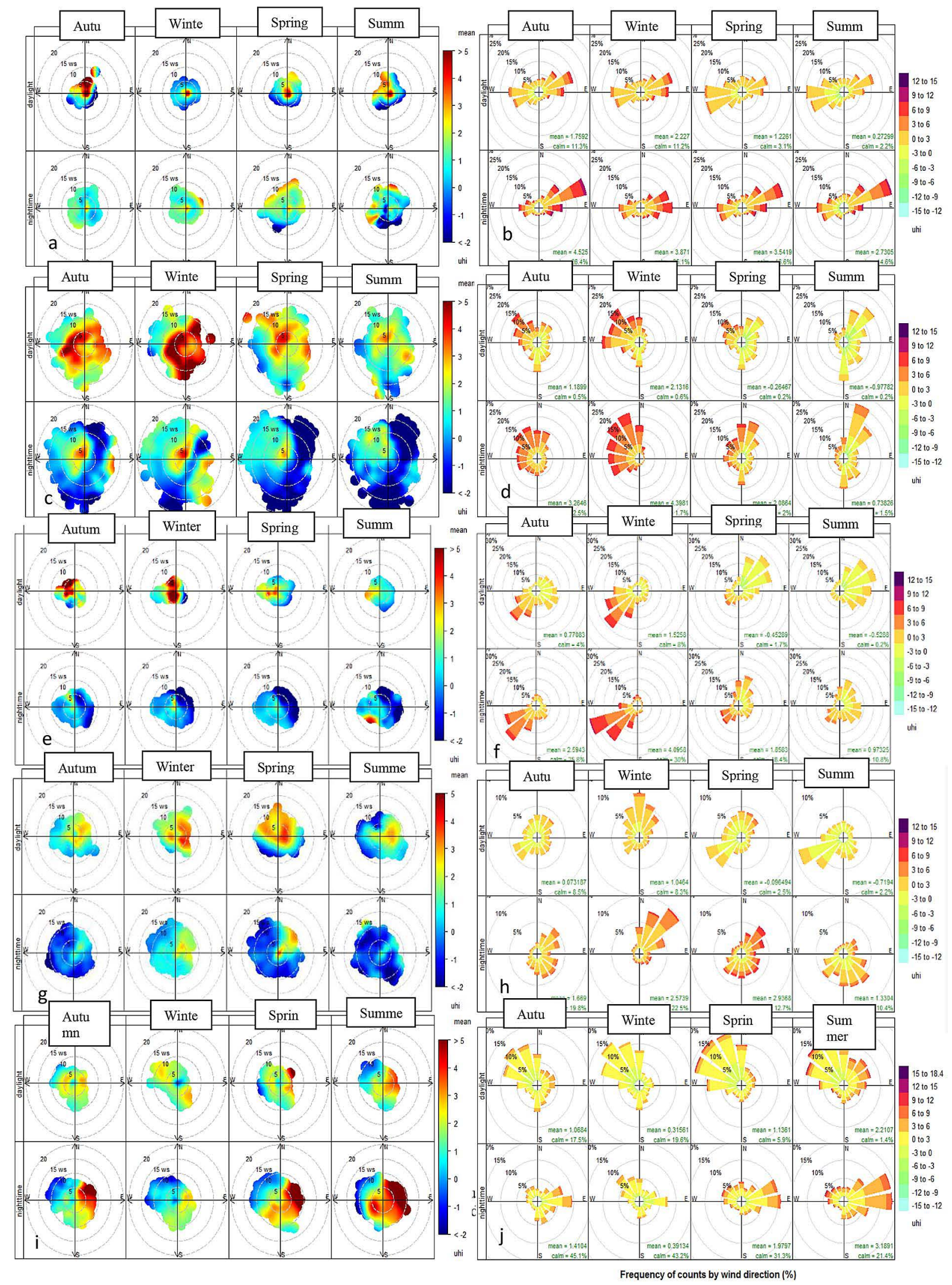

Figure 4. The effect of wind direction and wind speed on the diurnal UHIs in different seasons ((a and b: Melbourne; c and d: Sydney; e and f: for of Brisbane; $g$ and h: Adelaide; and i and j: Canberra) 
that the daytime and night time UHI intensity are strongest in winter and weakest in summer. The highest nocturnal UHI intensities are linked with lower wind speeds in all seasons (Figure 4e, f), low to high wind speeds from the southwest, northwest or west are associated with the strongest positive daytime UHI intensities in all seasons. The winds from the east or southeast are related with lower daytime UHI intensities. The highest positive night time UHI intensities were observed at lower wind speeds (wind speeds $\left.<1 \mathrm{~m} \cdot \mathrm{s}^{-1}\right)$. The winds from the west, southwest or northwest yield higher positive night time UHI intensities in all seasons. The maximum negative night time UHI intensities appeared at high wind speeds when wind is blowing from the southwest or southeast. Figure 4g, h presents seasonal daytime and night time UHI intensities and their relation to wind speed and wind direction in the city of Adelaide, negative relationships between wind speed and UHI intensity are generally observed in both the day and the night across all seasons. The results suggest that the mean daytime UHIs are generally higher than the mean night time UHIs under calm or low wind speed conditions during all seasons. The observations of the night time and daytime UHI with the daytime and night time wind data (wind speed and wind direction), show that the highest night time UHI intensities occur under calm to low wind speeds when wind blows from the east or northeast during all seasons. In contrast, higher daytime UHI intensities were observed when winds originated from the east or southeast under calm to light wind speed conditions in summer and autumn. However, in the spring and winter seasons, high positive daytime UHI intensities occur for all wind data conditions. The results also show that the day time and night time UHI intensity tend to be highest in spring and lowest in autumn for calm conditions. UCIs were more frequent during the night than the day, especially in hot seasons (spring and summer). It is interesting to note that, in all seasons, wind from all directions (except east or northeast) give lower or negative night time or daytime UHI intensities for all wind speeds, but these reach their peak at high or strong wind speeds. In contrast, in the city of Canberra (Figure 4i, j), for both day and night periods, lower UHI intensities corresponded with low wind speeds in all seasons. When the winds blow from the east or northeast, the UHI intensity could be as large as $3-4^{\circ} \mathrm{C}$ on summer days or $>5^{\circ} \mathrm{C}$ on summer, spring and autumn nights.
However, when winds originate from the southeast or southwest, high daytime UHI intensities at high wind speed conditions occur during winter and spring. In contrast, winds from the northwest or west favour higher negative UHI intensities at high wind speeds in all seasons. The maximum summer daytime UCI intensity is linked with northwesterly winds at wind speeds $>6 \mathrm{~m} \cdot \mathrm{s}^{-1}$, while the largest winter daytime UCI intensities were found at low wind speeds (wind speeds $<2 \mathrm{~m} \cdot \mathrm{s}^{-1}$ ). The prevailing day wind directions are from the north and northwest in all seasons, whereas winds originating in the east and northeast are common in all seasons during the night (except in winter).

\section{Regression analysis}

The final step in the wind analysis was regression statistical test. Figure 5 depicts the results of a linear regression analysis between the mean UHI intensity and wind speed in the urban region for each study cities. Regression results suggest that wind speed has considerable influence on UHI intensity with large wind speeds reducing UHI intensities. The linear regression coefficient showed a high correlation between UHI intensity and wind speed. This relationship was intensified in large cities, in particular, Sydney. A detailed analysis shows that for the Melbourne's urban site, a moderate negative relationship $(\mathrm{R} 2=0.77$ ) between the UHI intensity and the wind speed, as one-unit increase in the wind speed $\left(+1 \mathrm{~m} \cdot \mathrm{s}^{-1}\right)$ could decrease average UHI intensity as much as $0.42^{\circ} \mathrm{C}$. However, for the Sydney's urban site $(\mathrm{R} 2=0.73)$, a one-unit increase in the mean wind speed $\left(+1 \mathrm{~m} \cdot \mathrm{s}^{-}\right.$ $\left.{ }^{1}\right)$ results in a decrease in average UHI intensity of $0.33^{\circ} \mathrm{C}$. While, the linear regression for UHI at the Brisbane's urban site against wind speed (R2 $=0.76$ ) showed that a one-unit increase in wind speed $\left(+1 \mathrm{~m} \cdot \mathrm{s}^{-1}\right)$ results in a reduction in average $\mathrm{UHI}$ intensity of $0.52^{\circ} \mathrm{C}$. The linear regression for UHI for the Adelaide urban site against wind speed resulted in a quite strong negative relationship (R2 $=0.97$ ) between them, with a one-unit increase in the wind speed $\left(+1 \mathrm{~m} \cdot \mathrm{s}^{-1}\right)$ decreasing average UHI intensity by $0.31^{\circ} \mathrm{C}$. Finally, for the urban site in the city of Canberra $(\mathrm{R} 2=0.55)$, the rate of decrease in UHI is approximately $0.2^{\circ} \mathrm{C}$ per $1 \mathrm{~m} \cdot \mathrm{s}^{-1}$ increase in wind speed. This is the lowest sensitivity to wind of all the cities included in this study. It should be mentioned that the results from these regressions that were all statistically significant at the $95 \%$ confidence level. 


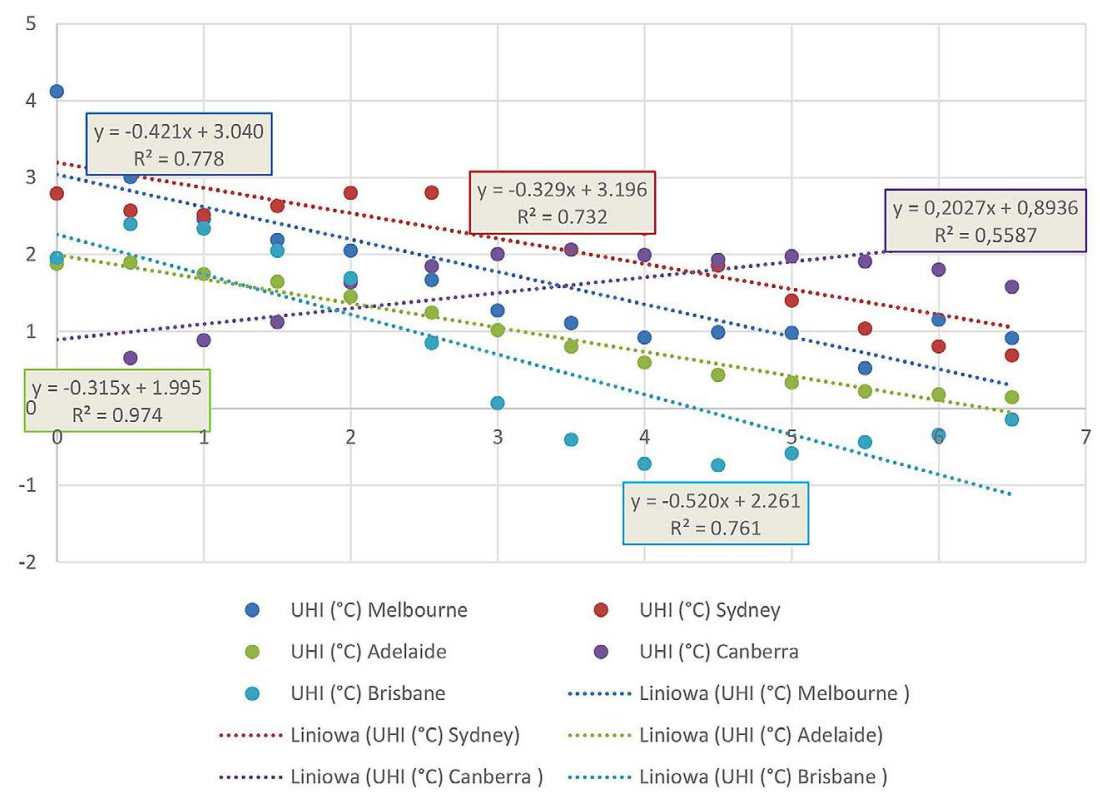

Figure 5. The mean UHI intensity for the ten categories of wind speed in all study cities

\section{DISCUSSION}

According to previous research, wind speed and wind direction have the potential to act as a strong control on heat island formation (Santamouris et al. 2017; Kim \& Baik 2005; Comrie 2000). Hence, a separate analysis was used to investigate the influence of wind speed and wind direction on UHI intensity in this study. The analysis was performed during the day, night and seasonal cycles, using hourly wind data retrieved from the surface weather stations (one urban station and one rural station) for each of five Australia cities. In general, in all cities, prevailing wind direction was shown to influence the UHI intensity. The most intense UHIs occurred when winds originated from drier or urban regions. Meanwhile, winds that originated from the sea were related with small or negative intensities of the UHI. In most cities, lower wind speeds were also shown to drive a strong heat island during both daytime and night-time cycles and in all seasons. The results show that the greatest UHI intensities are recorded when wind is weak (less than $2 \mathrm{~ms}^{-1}$ ), while the lowest magnitudes are found when wind speeds exceed $6 \mathrm{~m} \cdot \mathrm{s}^{-1}$. Further, the critical wind speed value, above which the strength of the UHI is considerably minimized, is around $4-5 \mathrm{~m} \cdot \mathrm{s}^{-1}$.

According to previous research, the prevailing wind direction may influence urban temperatures when the observation sites are situated in a mountain range (Comrie 2000) or close to the coastal line (Werner et al. 2000). In this study, the results suggest that wind direction has a significant impact on UHI intensity that was clear for both the diurnal and seasonal cycle and for winds that originated from a variety of geographic conditions (inland, coastal, urban, flat, mountainous etc.). For example, in the city of Melbourne winds from the seaside (southern or southeast or southwest) orientation can cool the urban area and reverse the usual thermal flux, thereby removing the UHI effect at wind speeds above $5 \mathrm{~m} \cdot \mathrm{s}^{-1}$. However, winds from the northern direction (inland) are mainly responsible for allowing the development of strong UHIs. Meanwhile, for Sydney and Brisbane, only winds from the west (drier area) correlated with the development of strong UHIs, whereas winds from the ocean side were mainly responsible for the development of UCIs, particularly at wind speeds above $4 \mathrm{~m} \cdot \mathrm{s}^{-1}$. For Adelaide, winds from the east or north increase the intensity of UHIs at all wind speeds, whilst winds from other directions help to cool the city. Finally, for Canberra (inland city), winds from the east or north intensify the UHI effect, while winds from the west may cool the urban area at high wind speeds.

The results of the linear regression analysis between the mean UHI intensity and mean wind speed magnitudes support the above results; mean UHI intensity exhibited significant negative correlations with wind speed, i.e., as wind speed increases the UHI magnitude decreases. The inverse association between wind speed and UHI 
strength can be explained by a rise in urban heat advection with greater wind speeds (e.g. Kim and Baik 2005). Numerous previous studies have examined the influence of wind speed on the characteristics of UHIs (Kim and Baik 2002; Morris, Simmonds and Plummer 2001; Santamouris et al. 2017; Schatz and Kucharik 2014). These studies report that UHIs are better formed under calm or low wind conditions, while higher wind speeds are correlated with small (or even negative) intensities of UHI which agrees with what was observed in this study. In contrast, studies carried out by Sofer and Potchter (2006) and Elsayed (2011) in Israel and Malaysia respectively, suggested that cooling from wind was not an important control on UHI intensity. These results are somewhat supported here in that in the city of Canberra, the results show that the UHI intensity did not increase meaningfully under calm conditions (wind speed equals zero), although UHI intensities were still shown to be lowest when wind speeds exceed about $2 \mathrm{~m} \cdot \mathrm{s}^{-1}$. Hence, some of the discrepancies between previous studies may have to do with local geographic differences between cities that impact on the sensitivity of UHI to wind. Unlike most previous studies, the statistical analysis presented in this study revealed that both wind speed and wind direction play a key role in UHI development at different times of day. Although higher average nocturnal UHI intensities were observed in most cities, on average, stronger UHI intensities were recorded during the daytime (than the night-time) under calm weather conditions (wind speed equals zero) in all cities (except Canberra, where calm night time had greater UHI intensities than calm day time in all seasons). However, most previous studies for other cities reported that the greatest night-time UHI occurs under calm conditions, suggesting that higher wind speeds alter the cooling rates in the rural and urban regions and modify night-time UHI strength (Kolokotsa, Psomas and Karapidakis 2009; Erell and Williamson 2007; Eliasson 1996). Polar plots and wind roses of the wind speed and direction relative to UHI intensities are depicted for the various correlations between each category of wind speed and wind direction during both day and night periods and/ or during all seasons. These plots enable a clear visual presentation of the least to most favourable wind (wind speed and wind direction) conditions for UHI development. In all cities (except Canberra), the largest UHI intensities were typically associated with low to calm weather conditions, while higher wind speeds are associated with small UHI intensities for both day time and nighttime in all seasons. The seasonal UHI intensity also has a noticeable correlation with wind conditions. In the city of Melbourne, calm winter winds resulted in higher UHI intensities than calm summer winds for both day and night periods. On the other hand, calm spring winds had greater UHI intensities than calm summer winds for both day and night periods. In the cities of Brisbane and Sydney, winter UHIs tended to be higher than summer UHIs under calm conditions. Conversely, in Canberra, wind direction was more important than wind speed in determining UHI intensity although calm summer winds still had higher UHI intensities than calm winter winds for both day and night periods. In built-up areas, more incoming solar radiative energy is absorbed during the day time than can be quickly emitted by nighttime radiative cooling, which delays the decrease of air temperature. In contrast, in non-urban regions, nocturnal radiative cooling happens more quickly and an intense night-time inversion layer forms. The contrast in surface energy balance between urban and non-urban environments can help explain the strong UHI development under favourable weather conditions (calm weather conditions). In windy condition, however, turbulent as well as radiative energy exchanges at the ground surface which helps to accelerate the loss of heat from urban areas which tends to damp the UHIU intensity (Kim and Baik 2002).

\section{CONCLUSION}

In this study the wind speed and direction, have been explored to determine how local wind conditions impact on UHI intensity in and around the study cities. By using hourly data over the study period, the relationship between the influences of wind data on the urban heat island intensity was examined for annual, seasonal, monthly and daily data. This approach is novel in that previous studies on the links between wind data and UHI intensity have focussed expressly on atypical weather conditions or have included or short-term data sets. This study also pioneers a new method of investigating the links between wind speed and direction and UHI intensity by combining wind roses and polar plots embedded with UHI intensity data. Using this approach, this study was able to identify which wind speeds and 
direction corresponded (on a diurnal, daily, seasonal and annual basis) to the highest and lowest UHI intensities in a way that no previous studies have been able to do. These results revealed that there is a strong inverse relationship between wind speed and UHI intensity across all cities. However, it also showed that wind direction has at least as much influence on UHI intensity as wind speed in most cities. In terms of wind direction, this study revealed that UHI intensities were highest when winds originated from dry inland regions and were lowest when winds originated from the sea or higher and wetter parts of the landscape. In many cities, it was also found that wind direction could have a stronger influence on UHI intensity than wind speed with strong winds that originated from dry inland areas still resulting in very high UHI intensities. This is the first time that this observation has been made in the scientific literature.

\section{Acknowledgments}

I would like to thank Ministry of High Education and Scientific Research - Mustansiriyah University and Australian Government Research Training Program Scholarship to fund this search.

\section{REFERENCES}

1. Comrie A.C. 2000. Mapping a Wind-Modified Urban Heat Island in Tucson, Arizona (with Comments on Integrating Research and Undergraduate Learning). Bulletin of the American Meteorological Society, 81(10), 2417-2432.

2. Eliasson I. 1996. Urban nocturnal temperatures, street geometry and land use. Atmospheric Environment, 30(3), 379-392.

3. Elsayed I.S.M. 2012. Effects of Population Density and Land Management on the Intensity of Urban Heat Islands: A Case Study on the City of Kuala Lumpur, Malaysia. in: BM Alam (ed.) Application of Geographic Information Systems, InTech, Rijeka, 14.

4. Erell E., Williamson T. 2007. Intra-urban differences in canopy layer air temperature at a mid-latitude city. International Journal of Climatology, 27(9), 1243-1256.

5. Harlan S.L., Brazel A.J., Prashad L., Stefanov W.L., Larsen L. 2006. Neighborhood microclimates and vulnerability to heat stress. Social Science \& Medicine, 63(11), 2847-2863.

6. Kenney W.L., Craighead D.H., Alexander L.M.
2014. Heat waves, aging, and human cardiovascular health. Med. Sci. Sports Exerc., 46, 1891-1899.

7. Kim Y.-H., Baik J.-J. 2004. Daily maximum urban heat island intensity in large cities of Korea. Theoretical and Applied Climatology, 79(3-4), 151-164.

8. Kim Y.-H., Baik J.-J. 2002. Maximum urban heat island intensity in Seoul. Journal of Applied Meteorology, 41(6), 651-659.

9. Kim Y.-H., Baik J.-J. 2005. Spatial and temporal structure of the urban heat island in Seoul. Journal of Applied Meteorology, 44(5), 591-605.

10. Knowlton K., Lynn B., Goldberg R.A. 2007. Projecting heat-related mortality impacts under a changing climate in the New York City region. Journal of Public Health, 97, 2028-2034.

11. Kolokotsa D., Psomas A., Karapidakis E. 2009. Urban heat island in southern Europe: The case study of Hania, Crete. Solar Energy, 83(10), 1871-1883.

12. Li L.-G., Liang Z.-B., Wang H.-B., Li C.-J., Wang X.-Y., Zhao X.-L. 2011. Urban heat island characteristics in Shenyang under different weather conditions [J]. Transactions of Atmospheric Sciences, $1,10$.

13. Morris C., Simmonds I., Plummer N. 2001. Quantification of the influences of wind and cloud on the nocturnal urban heat island of a large city. Journal of Applied Meteorology, 40(2), 169-182.

14. Morris C., Simmonds I. 2000. Associations between varying magnitudes of the urban heat island and the synoptic climatology in Melbourne, Australia. International Journal of Climatology, 20(15), 1931-1954.

15. Oke T.R. 1982. The energetic basis of the urban heat island. Quarterly Journal of the Royal Meteorological Society, 108(455), 1-24.

16. Prafull S., Noyingbeni K., Pradipika V. 2017. Impact of land use change and urbanization on urban heat island in Lucknow city, Central India. A remote sensing based estimate. Journal of sustainable cities and society, 32, 100-114.

17. Team R.C. 2019. R: A Language and Environment for Statistical Computing [Computer Software], Foundation for Statistical Computing, R Vienna, Austria.

18. Sani S. 1990. Urban climatology in Malaysia: An overview', Energy and Buildings, 15(1), 105-117.

19. Santamouris M., Haddad S., Fiorito F., Osmond P., Ding L., Prasad D., Zhai X., Wang R. 2017. Urban Heat Island and Overheating Characteristics in Sydney, Australia. An Analysis of Multiyear Measurements. Sustainability, 9(5), 712.

20. Santamouris M. 2015. Analyzing the heat island magnitude and characteristics in one hundred Asian 
and Australian cities and regions. Science of The Total Environment, 512, 582-598.

21. Schatz J., Kucharik C.J. 2014. Seasonality of the Urban Heat Island Effect in Madison, Wisconsin. Journal of Applied Meteorology and Climatology, 53(10).

22. Shashua-Bar L., Hoffman M. 2000. Vegetation as a climatic component in the design of an urban street: An empirical model for predicting the cooling effect of urban green areas with trees. Energy and Buildings, 31(3), 221-235.

23. Sofer M., Potchter O. 2006. The urban heat island of a city in an arid zone: the case of Eilat, Israel. Theoretical and Applied Climatology, 85(1), 81-88.
24. Yong-jiang Y. 2009. Study on the Relationship between Meteorological Condition and Urban Heat Island Effect in Fuzhou. Journal of Anhui Agricultural Sciences, 3, 104.

25. Wickham H., François R., Henry L., Müller K. 2021. Dplyr: A Grammar of Data Manipulation. Rpackage version 1.0.6. https://CRAN.R-project. org/package $=$ dplyr. [89] Just M.A., Carpenter P.A.T.

26. Zhong S., Qian Y., Zhao C., Leung R., Wang H., Yang B., Fan J., Yan H., Yang X.-Q., Liu D. 2017. Urbanization-induced urban heat island and aerosol effects on climate extremes in the Yangtze River Delta region of China', Atmos. Chem. Phys., 17, 54395457. https://doi.org/10.5194/acp-17-5439-2017. 